\title{
Design and Analysis of a Wide Stopband Microstrip Dual-band Bandpass Filter
}

\author{
Salah I. Yahya ${ }^{1,2}$, Abbas Rezaei ${ }^{3}$, Yazen A. Khaleel ${ }^{2}$ \\ ${ }^{1}$ Department of Communication and Computer Engineering, Cihan University-Erbil, Erbil, \\ Kurdistan Region - F.R. Iraq \\ ${ }^{2}$ Department of Software Engineering, Faculty of Engineering, Koya University, \\ Koya KOY45, Kurdistan Region - F.R. Iraq \\ ${ }^{3}$ Department of Electrical Engineering, Kermanshah University of Technology, \\ Kermanshah, Iran
}

\begin{abstract}
A novel configuration of a dual-band bandpass filter (DB-BPF) working as a harmonic attenuator is introduced and fabricated. The proposed filter operates at $3 \mathrm{GHz}$, for ultrahigh-frequency and super high-frequency applications, and 6.3 GHz, for wireless applications. The presented layout has a symmetric structure, which consists of coupled resonators. The designing of the proposed resonator is performed by introducing a new $L C$ equivalent model of coupled lines. To verify the $L C$ model of the coupled lines, the lumped elements are calculated. The introduced filter has a wide stopband up to $85 \mathrm{GHz}$ with $28^{\text {th }}$ harmonic suppression, for the first channel, and $13^{\text {th }}$ harmonic suppression, for the second channel. The harmonics are attenuated using a novel structure. Furthermore, the proposed BPF has a compact size of $0.056 \lambda_{\mathrm{g}}{ }^{2}$. Having several transmission zeros that improve the performance of the presented BPF are another feature. The proposed DB-BPF is fabricated and measured to verify the design method, where the measurement results confirm the simulations.
\end{abstract}

Index Terms-Microstrip; bandpass filter; dual band; harmonic attenuator; ultra-high frequency; super high frequency.

\section{INTRODUCTION}

Recently, microstrip devices such as microstrip bandpass filters (BPFs) and diplexers have been widely used in many applications (Rezaei and Yahya, 2019; Yahya, Rezaei and Nouri, 2020a; 2020b; 2020c; Rezaei, Yahya and Jamaluddin, 2020; Rezaei, et al., 2020;). High-performance microstrip dual-band BPFs (DB-BPFs) with planar structures have been used widely in multiband microwave/RF transceivers. Several kinds of DB-BPFs are reported in Hayati and Noori (2011; 2012); Liu, Li and Ren (2013); Sun, et al. (2013); Chen, et al. (2014); Huang and Huang (2014); Pan, et al. (2015);

ARO-The Scientific Journal of Koya University

Vol. IX, No.2 (2021), Article ID: ARO.10908, 8 pages

DOI: 10.14500/aro.10908

Received: 21 November 2021; Accepted: 04 December 2021 口内

Regular research paper: Published: 15 December 2021

Corresponding author's email: salah.ismaeel@koyauniversity.org

Copyright (C) 2021 Salah I. Yahya, Abbas Rezaei, Yazen A. Khaleel.

This is an open access article distributed under the Creative

Commons Attribution License.
Wu, et al. (2015); Ogbodo, Wang and Yeo (2016); Malherbe (2016); Zhu and Abbosh (2016); Xu and Zhu (2017); Rezaei and Noori (2017); Rezaei, et al. (2019); Rezaei and Yahya (2019); Challal, Hocine and Mermoul (2019); Moitra, Dey and Bhowmik (2019); Borjlu and Khadem (2019); Khani, Danaie and Rezaei (2019); Karimi, et al. (2019); Oudaya and Tamilselvan (2020); Li, Kang and Liu (2020); and Rezaei, et al. (2020). A quad-mode resonator with stub-to-stub coupling structure in Sun, et al. (2013), multimode steppedimpedance resonators in Chen, et al. (2014), radial stubs and lumped capacitors in $\mathrm{Xu}$ and $\mathrm{Zhu}$ (2017), complex spiral resonators in $\mathrm{Wu}$, et al. (2015), E-shape resonator in Liu, Li and Ren (2013), and coupled open loops in Hayati and Noori (2011; 2012); Rezaei and Noori (2017) are all adopted to obtain DB-BPFs. However, these DB-BPFs could not improve the return loss, where the best-achieved return loss is $-21 \mathrm{~dB}$. Since the harmonic attenuation is a significant factor, a welldesigned filter must suppress harmonics. Nevertheless, only the designers in Sun, et al. (2013) were able to suppress the $8^{\text {th }}$ harmonic. The DB-BPFs in Chen, et al. (2014); Xu and Zhu (2017); Wu, et al. (2015); and Liu, Li and Ren (2013) could suppress just the first harmonic, which is a weakness of their works. Meanwhile, Hayati and Noori $(2011 ; 2012)$ could attenuate the fourth and second harmonics, respectively. In Rezaei and Noori (2017), the second harmonic is attenuated below $-20 \mathrm{~dB}$. In Ogbodo, Wang and Yeo (2016), several coupled U-shape resonators are connected together to create two close passbands at $1.74 \mathrm{GHz}$ and $1.87 \mathrm{GHz}$. Meanwhile, it has large insertion losses without harmonic attenuation. In Zhu and Abbosh (2016), embedded coupled lines have been used to provide a high selectivity DB-BPF with two passbands from $2.28 \mathrm{GHz}$ to $2.67 \mathrm{GHz}$ and $3.35 \mathrm{GHz}$ to 3.63 GHz for WLAN and WiMAX applications, respectively. However, it could only suppress $2^{\text {nd }}$ harmonic. The dual-band frequency response in Malherbe (2016) has been created by employing cascaded shunt open and short steps. In Pan, et al. (2015), a tri-mode resonator with varactors has been used to obtain a dual-band frequency response whereas it needs a discriminated coupling to suppress the unwanted mode. In Huang and Huang (2014), a large size dual-mode defected stub-loaded resonator provides a high selectivity passband. In 
Oudaya and Tamilselvan (2020), a conductor-backed CPWbased DB-BPF with a compact size has been presented for satellite applications. An adjustable high-selectivity DB-BPF is designed in Li, Kang and Liu (2020) using a quantic-mode resonator. In Challal, Hocine and Mermoul (2019), a novel DB-BPFs for wireless communication systems has been presented. In Moitra, Dey and Bhowmik (2019), the design and analysis of a DB-BPFs using coupled line structures, DGS, and series inductive metallic vias have been presented. A quad-section DB-BPF has been designed in Borjlu and Khadem (2019) using a radial stub for wireless applications. In Khani, Danaie and Rezaei (2019), a microstrip DB-BPF with compact size and wide bandwidth has been introduced. A compact DB-BPF with an ultra-narrow band has been proposed in Karimi, et al. (2019) for WiMAX applications. For providing an alternative method to miniaturize a microstrip DB-BPF, a symmetrical fractal structure has been utilized in Lu, et al. (2017). Two DB-BPFs operated at 2.4/5.7 GHz and $0.9 / 1.575 \mathrm{GHz}$ have been proposed in Yang, et al. (2010) for WLANs and GPS, respectively. In Wu, et al. (2008), a microstrip DB-BPF with low selectivity has been introduced based on quarter-wavelength resonators for WLAN and GPS applications. A microstrip DB-BPF using tri-section stepped impedance and short stub-loaded stepped impedance resonators has been designed in $\mathrm{Li}$, et al. (2015) for WLAN and WiMAX applications. In Hammed (2015), an E-shape microstrip structure has been used to design a DB-BPF for multiband wireless communication systems. To achieve a microstrip DB-BPF for WLAN applications in Zong, et al. (2012), a microstrip embedded scheme resonator has been utilized. Similar to the other DB-BPFs mentioned above, the works in Malherbe (2016); Pan, et al. (2015); Huang and Huang (2014); Lu, et al. (2017); Yang, et al. (2010); Wu, et al. (2008); Li, et al. (2015); Hammed (2015); and Zong, et al. (2012) could not improve stopband properties. In Hayati and Noori (2011), a microstrip DB-BPF has been reported which could attenuate $4^{\text {th }}$ harmonic. It does not have a novel structure because of using two stub-loaded open-loop resonators. Two single-band BPFs with high losses and large dimensions are designed in Feng, Gao and Che (2015); Salleh, et al. (2008) using similar basic structures. The proposed filter in Salleh, et al. (2008) consists of two meandered closed loops but Feng, Gao and Che (2015) used only a simple loop. They used closed loops consisting of coupled lines but they could not suppress the harmonics well. Using SIRs (interlocked stepped-impedance resonators), a DB-BPFs with small size is presented in Hsu, Lyu and Chang (2020) to work at 900-1000 $\mathrm{MHz}$ and $1427-1518 \mathrm{MHz}$ bands. In Badiaa, et al. (2020), a novel multiband filter has been presented using open ring and stubs resonators.

This paper presents a novel compact DB-BPF with remarkable features such as $13^{\text {th }}$ harmonic attenuation, high selectivity at both passbands, high isolation between two channels, and good return losses better than $35 \mathrm{~dB}$. The designing process is prepared as follows: At the first, a novel dual-mode resonator using coupled lines is introduced. Then, an approximated equivalent $L C$ model for coupled lines is proposed. Next, an equivalent $L C$ circuit of the introduced resonator is presented by utilizing the $L C$ model of coupled lines. To verify the proposed $L C$ circuit, the lumped inductors are calculated.

Moreover, the ranges of coupling and open-end capacitors are estimated and optimized. The simulation results of the $L C$ model and EM simulator are compared with each other to verify the proposed model. After that, a theoretical method is presented to attenuate the harmonics. Finally, a DB-BPF is introduced using the proposed resonator.

\section{Design of a Novel Resonator}

The layout configuration of the basic resonator used in this study is shown in Fig. 1a. It has a symmetrical structure consisting of two L-shape coupled lines combined by a transmission line with the physical length $l_{\mathrm{S} 1}$. The vertical and horizontal coupled lines, which form the L-shaped coupled lines, have the exact physical dimensions. To obtain an equivalent $L C$ circuit, first, we proposed an $L C$ model of coupled lines as indicated in Fig. 1b. The proposed $L C$ model is not the exact model because the number of inductors and capacitors in the exact model will be increased significantly. Therefore, we represented the equivalent model of a transmission line only by an inductance. Four inductors (presented by $L_{\mathrm{c}}$ in Fig. 1b) model the coupled lines, where each $L_{\mathrm{c}}$ is related to a half of a line.

Moreover, three capacitors of $C_{\mathrm{c}}$ show the coupling between lines. Fig. 1c demonstrates the $L C$ equivalent model of Fig. 1a. The vertical and horizontal coupled lines are replaced by the proposed $L C$ circuit of the coupled lines (Rezaei and Noori, 2017). The inductor $L_{\mathrm{S} 2}$ is related to the stubs with the physical lengths $l_{S 2}$. The capacitors $C_{\mathrm{o}}$ present the effect of open ends.

Fig. 1c shows the approximate equivalent circuit. Since the bents effects are significant at above $10 \mathrm{GHz}$, we ignored them in the $L C$ equivalent circuit. Meanwhile, the $L C$ equivalent model of coupled lines is an approximated circuit. To calculate the lumped inductors' values, we assume $l_{S 1}=1 \mathrm{~mm}, l_{S 2}=l_{c}=8 \mathrm{~mm}, S=0.2 \mathrm{~mm}$, and $w=0.8 \mathrm{~mm}$.

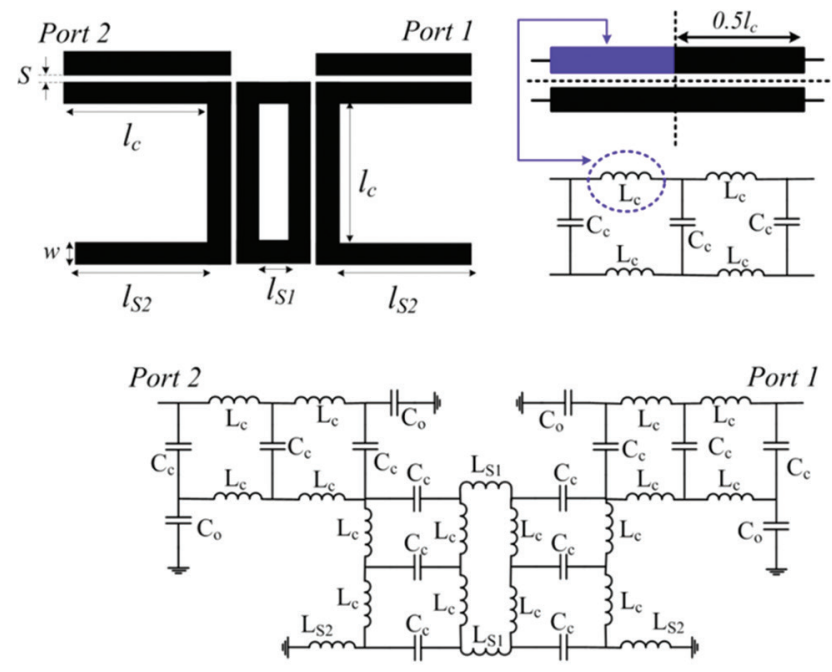

Fig. 1. (a) Proposed resonator, (b) proposed LC circuit of coupled lines, and (c) LC equivalent model of Fig. 1a. 
The width value of the line with length $l_{\mathrm{S} 1}$ is $0.8 \mathrm{~mm}$. This resonator is simulated for a substrate with $\varepsilon_{\mathrm{r}}=2.2$ and $h=0.7874 \mathrm{~mm}$. The EM simulation results show that it operates at $4.43 \mathrm{GHz}$. For $\mathrm{w} / h>1$, the characteristic impedance of lumped inductors $\left(Z_{\text {in }}\right)$ can be obtained (Hong and Lancaster, 2001). The characteristic impedance and effective dielectric constant are calculated as $Z_{\mathrm{c}}=94.14 \Omega$ and $\varepsilon_{\mathrm{re}}=1.76$ for all units of the proposed resonator with the width $0.8 \mathrm{~mm}$. The values of lumped inductors are calculated, according to Hong and Lancaster (2001), as $L_{\mathrm{c}}=0.85 \mathrm{nH}$, $L_{\mathrm{S} 1}=1.82 \mathrm{nH}$, and $L_{\mathrm{S} 2}=0.21 \mathrm{nH}$. Due to the features of coupling, the coupling capacitor $\left(C_{\mathrm{c}}\right)$ is chosen as a small value. We know that a capacitor can be created between two coupled lines which its value is proportional to the thickness of metal and length of lines. On the other hand, the coupling capacitor value has inverse coordination with the distance between lines. Since the thickness of metal is very small, and due to having small dielectric constant, the coupling capacitor value is in $\mathrm{fF}$. On the other hand, the open-end capacitor can be increased by increasing the ratio $\Delta L \sqrt{\varepsilon_{r e}} / Z_{c}$ where $\Delta L$ is an equivalent length of the transmission line (Hong and Lancaster, 2001). Due to computational complexity, we prefer to use an optimization method. As mentioned above, the values of inductors are already calculated and the range of capacitors values can be estimated in accordance with their equations. Therefore, we can change these capacitors values until the simulation results of the $L C$ model approach the EM simulations results. As shown in Fig. 2, three cases of capacitors values are used to show our optimization process. Figs. 2a-c show the comparison between the EM and $L C$ model results for $\left(C_{\mathrm{c}}=345 \mathrm{fF}, C_{\mathrm{o}}=2.8 \mathrm{pF}\right),\left(C_{\mathrm{c}}=280\right.$ $\left.\mathrm{fF}, C_{\mathrm{o}}=2.8 \mathrm{pF}\right)$, and $\left(C_{\mathrm{c}}=150 \mathrm{fF}, C_{\mathrm{o}}=0.3 \mathrm{pF}\right)$, respectively. These capacitors are obtained using an optimization method (the coupling capacitors values are assumed to be small). Figs. 2a-c verify both the proposed $L C$ model and calculated values. Due to the mentioned approximations, the EM and $L C$ simulated results are not completely overlapped. As explained above, some approximations are used due to the complexity of the $L C$ circuit, because our aim, in general, is to detect the effects of various parameters on the frequency response. Then, we can use the obtained results to optimize the main frequency response of our filter. On the other hand, the values of capacitors are obtained using an optimization method. Therefore, the values of capacitors are not exact. Fig. 2 shows the optimization process, which includes appropriate and inappropriate values. However, the dualmode features can be seen clearly.

\section{HaRmonic Attenuation Method}

Our harmonic attenuation technique is based on calculating resonance frequencies. Since our aim is to obtain a DB-BPF, the first and second calculated resonance frequencies are desired. If there is another resonance frequency, in addition to the main frequencies, it is an undesired harmonic. For removing this harmonic, first, we have to calculate the
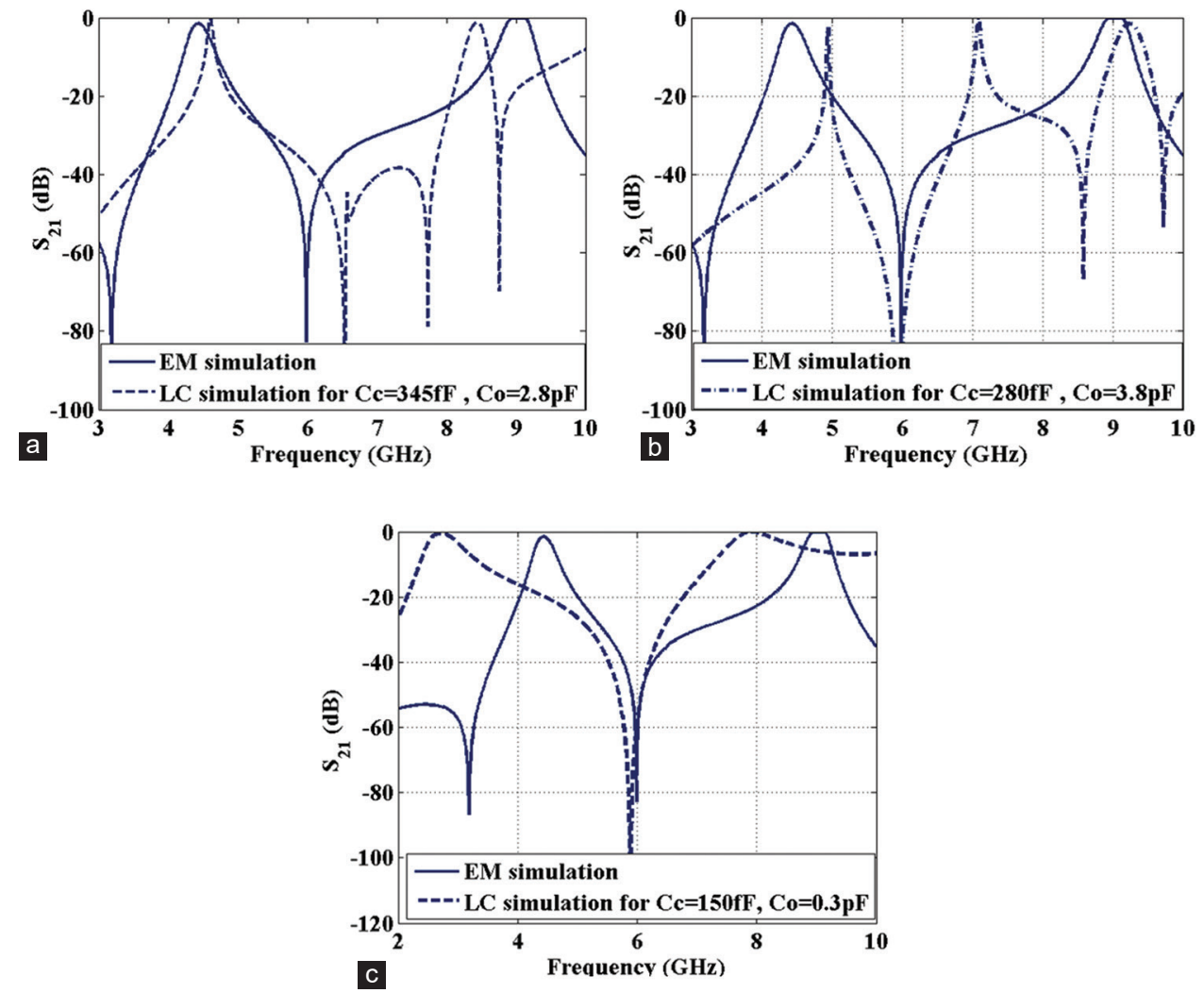

Fig. 2. Comparison between the EM and $L C$ simulations results for (a) $C_{\mathrm{c}}=345 \mathrm{fF}$ and $C_{\mathrm{o}}=2.8 \mathrm{pF}$ (b) $C_{\mathrm{c}}=280 \mathrm{fF}$ and $C_{\mathrm{o}}=3.8 \mathrm{pF}$ (c) $C_{\mathrm{c}}=150 \mathrm{fF}$ and $C_{\mathrm{o}}=0.3 \mathrm{pF}$. 
resonance frequencies. The resonance frequencies can be found when input admittance or input impedance is equal to zero. To calculate the input impedance, an equivalent model of the $L C$ circuit can be used. Therefore, an equivalent model of the proposed resonator is demonstrated in Fig. 3a, where $Z_{\mathrm{c}}$ is the characteristic impedance of coupled lines with the physical length $l_{c}$. Fig. 3b shows a simplified model of Fig. 3a, which is obtained by star-delta transformation for:

$$
\begin{aligned}
& Z a=\frac{j \omega L s 1\left(2 Z j Z k+Z_{k}^{2}\right)}{j \omega L s 1 Z j\left(2 Z j Z k+Z_{k}^{2}\right)} \\
& Z b=Z c+j \omega L_{s 2}\left(2 L_{s 2}+L_{s 1}\right)
\end{aligned}
$$

Where:

$$
Z j=\frac{j \omega L_{s 2}^{2}}{2 L_{s 2}+L_{s 1}} \text { and } Z k=Z c+\frac{j \omega L_{s 2} L_{s 1}}{2 L_{s 2}+L_{s 1}}
$$

Where, $\omega$ is the angular frequency. For obtaining the resonance frequencies, the input impedance (or input admittance) of the equivalent circuit should be zero. Therefore, in the resonance modes, an open circuit and a short circuit for the even and odd modes should be created, respectively. The input impedance can be calculated from the equivalent circuit presented in Fig. $3 \mathrm{~b}$. The impedance $Z_{\mathrm{a}}$ acts as an open circuit due to its position in the equivalent circuit. After calculating the input admittance and setting it to zero, another condition of resonance modes can be obtained as $Z_{\mathrm{b}}+Z_{\mathrm{c}}=0$. For simplifying in a symmetrical model, we can connect the same potential nodes. Equality of $Z_{\mathrm{b}}+Z_{\mathrm{c}}=$ 0 presents the odd mode resonance condition obtained by calculating the input impedance and applying the short circuit condition. Therefore, the even and odd resonance modes for $\omega_{\mathrm{e}}$ and $\omega_{\mathrm{o}}$ (angular resonance frequencies) can be written as:

even mode: $\mathrm{j} \omega_{e} L_{s 1} Z_{j}+2 Z_{j} Z_{k}+Z_{k}^{2}$

Odd mode: $2 Z_{c}+\mathrm{j} \omega_{o} L_{s 2}\left(2 L_{\mathrm{s} 2}+L_{s 1}\right)=0$

Equations (2.a) and (2.b) are obtained from the input impedance of the equivalent model presented in Fig. $3 \mathrm{~b}$. When we set the input impedance $Z_{\text {in }}=0$, the odd mode condition is obtained as shown in Equation (2.b). Furthermore, for 1/ $Z_{\text {in }}=0, \omega_{\mathrm{e}}$ (even mode resonance frequency) is achieved. In Equation (2.a), $Z_{\mathrm{j}}$ and $Z_{\mathrm{k}}$ should be calculated at $\omega=\omega_{\mathrm{e}}$. It can

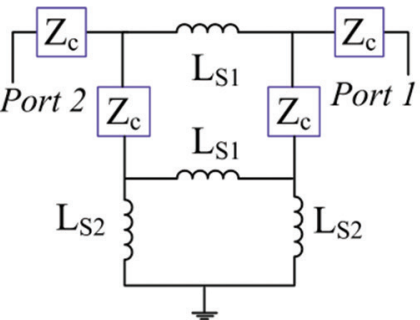

(a)

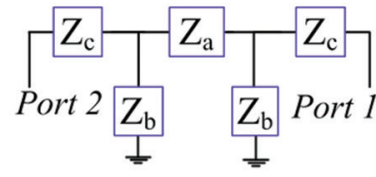

(b)
Fig. 3. (a) An equivalent model of our resonator and (b) a simplified model of Fig. 3a. be seen that there are several $\omega_{\mathrm{e}}$, which satisfy Equations (2.a) and (2.b). Two achieved angular resonance frequencies are fundamentals and the others are harmonics, which should be removed. To attenuate the harmonics, we assume that $\omega_{0}$ is the main resonance frequency. Since we have a dual-band response, an obtained $\omega_{\mathrm{e}}$ from Equation (2.a) is another main angular resonance frequency. By substituting $Z_{\mathrm{c}}$ in Equation (2.a) and solving Equation (2.a):

$$
\omega e=j Z c \frac{L s 2^{2}+L s 2 L s 1 \pm \sqrt{\left(L s 2^{2}+L s 2 L s 1\right)^{2}+1}}{2 L s 2^{2} L s 1}
$$

Where, $\omega_{\mathrm{e}}$ is a positive value thus according to the above discussion, there are not any harmonics.

\section{The Configuration of the Proposed DB-BPF}

Fig. 4 depicts the proposed DB-BPF layout (all dimensions are in $\mathrm{mm}$ ). It includes the introduced resonator loaded by the additional stubs, which are embedded to optimize the filter performance and miniaturize the circuit size. Each stub consists of two coupled closed rings. These rings shift the resonance frequencies to the left whereas they can save the normalized size (in $\lambda_{\mathrm{g}}{ }^{2}$ ). Moreover, increasing the number of these closed rings can improve the insertion and return losses. On the other hand, by removing these rings, the frequency selectivity will be decreased. Fig. 5 demonstrates the current

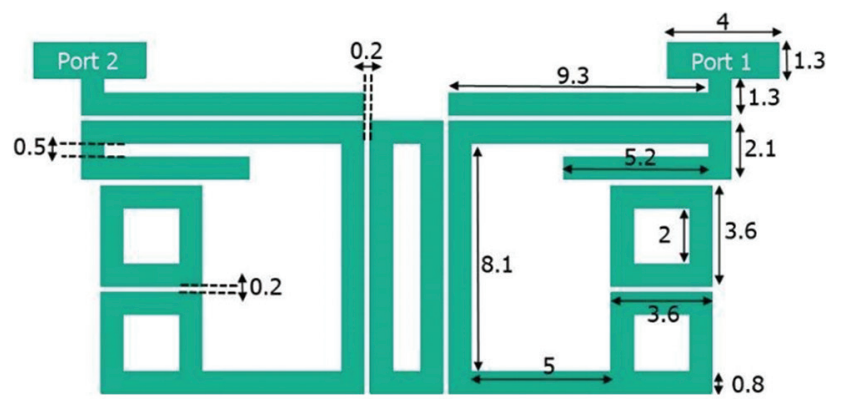

Fig. 4. Layout configuration of the presented dual-band bandpass filter.
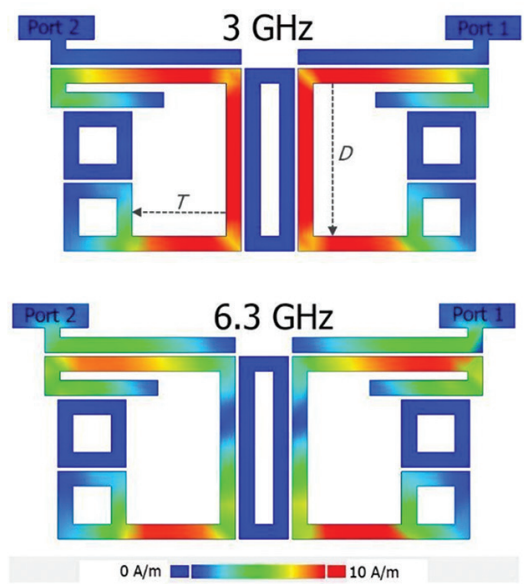

Fig. 5. Current density distributions at $3 \mathrm{GHz}$ and $6.3 \mathrm{GHz}$. 
density distributions over the proposed structure at $3 \mathrm{GHz}$ and $6.3 \mathrm{GHz}$. As shown in Fig. 5, the minimum current densities are in the coupled closed loops. As mentioned before, the coupled closed loops are added for optimization whereas they do not have a significant effect on creating the resonance frequencies. The maximum current densities are in the designed resonator. Fig. 6 shows the frequency responses as functions of the highlighted physical lengths. These lengths have the same effects on the quality of the frequency response. Hence, by tuning them, the best values are selected. The simulations are performed on a Rogers RT/ duroid5880 substrate with loss tangent $=0.0009, \varepsilon_{\mathrm{r}}=2.22$, and $h=31$ mil.

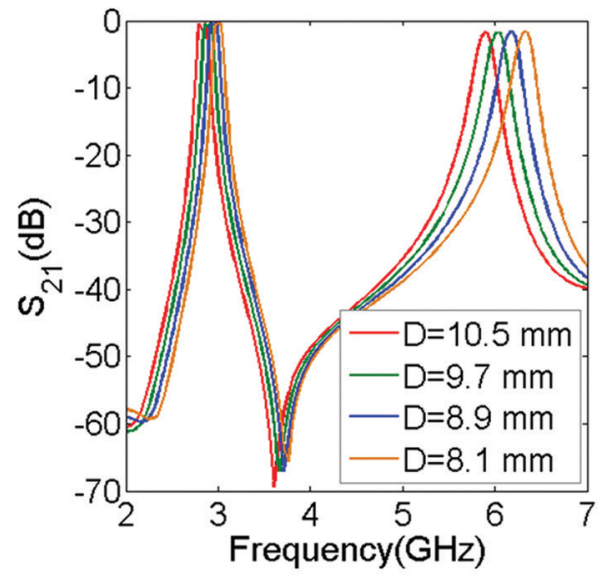

\section{Simulation and Measurement Results}

We used Advanced Design System software for simulating the proposed DB-BPF. We fabricated the proposed DB$\mathrm{BPF}$ on a Rogers RT/duroid5880 substrate with loss tangent $=0.0009, \varepsilon_{\mathrm{r}}=2.22$, and $h=31$ mil. A N5230A network analyzer is used to measure the fabricated filter. Fig. 7a illustrates the simulation and measurement results. The simulated narrowband frequency response in the range of 2.5 GHz-7 GHz is shown in Fig. 7b. At the first and second passbands, the $-3 \mathrm{~dB}$ cutoff frequencies are $(2.9 \mathrm{GHz}$, $3.15 \mathrm{GHz})$ and $(6.26 \mathrm{GHz}, 6.38 \mathrm{GHz})$, respectively, so that it operates at $3 \mathrm{GHz}$ and $6.3 \mathrm{GHz}$. Since the frequency of

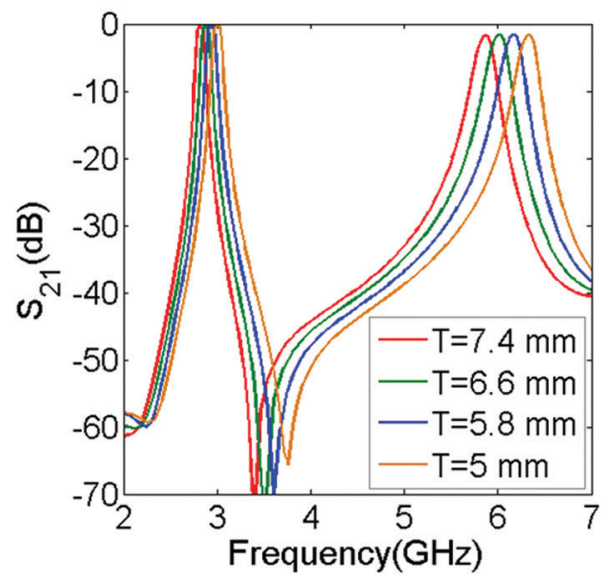

Fig. 6. Frequency responses as functions of $D$ and $T$ (shown in Fig. 5).
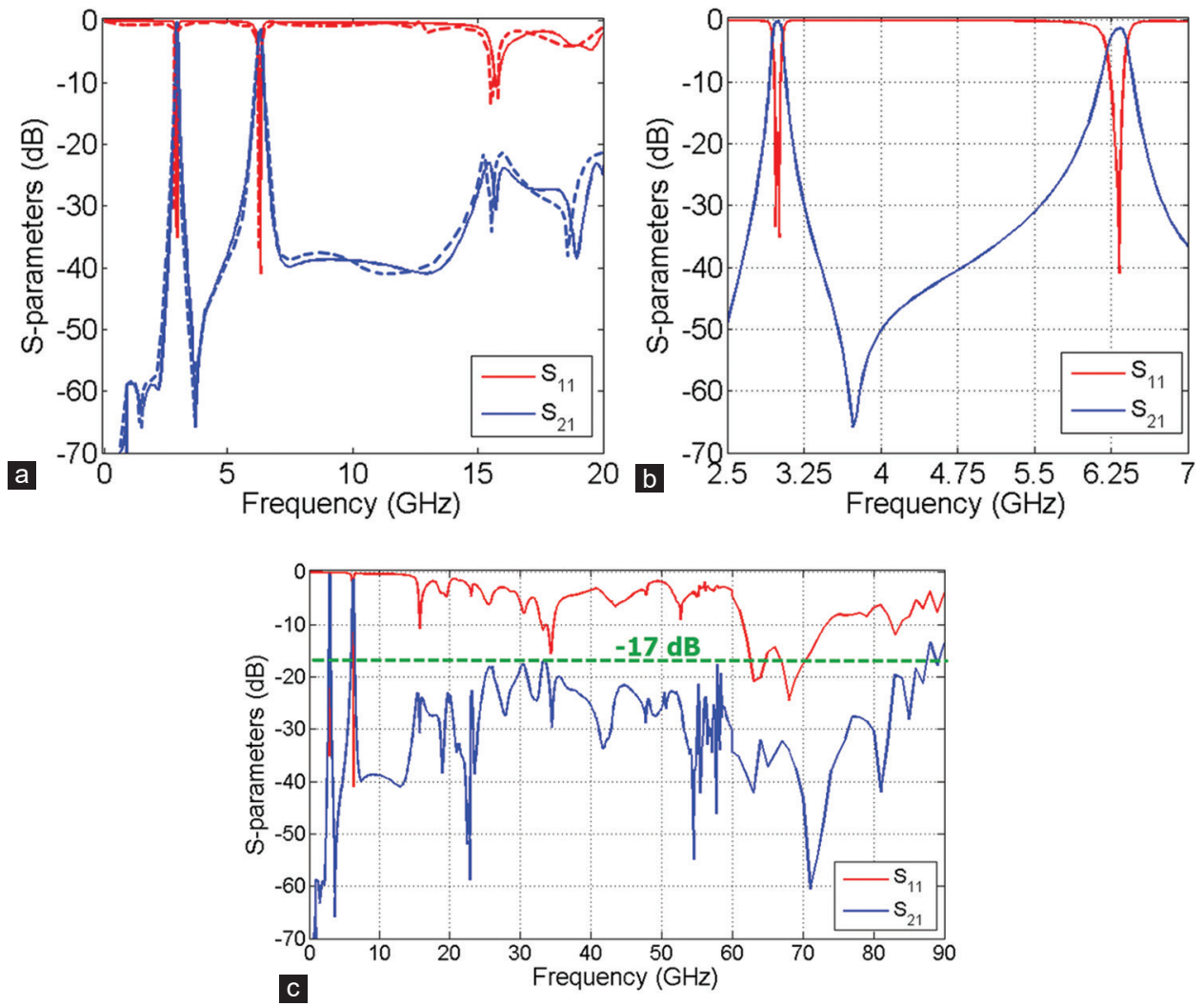

Fig. 7. Proposed dual-band bandpass filter results: (a) Measured (dashed line) and simulated (solid line) frequency responses, (b) simulated narrowband frequency response, and (c) simulated wideband frequency response. 


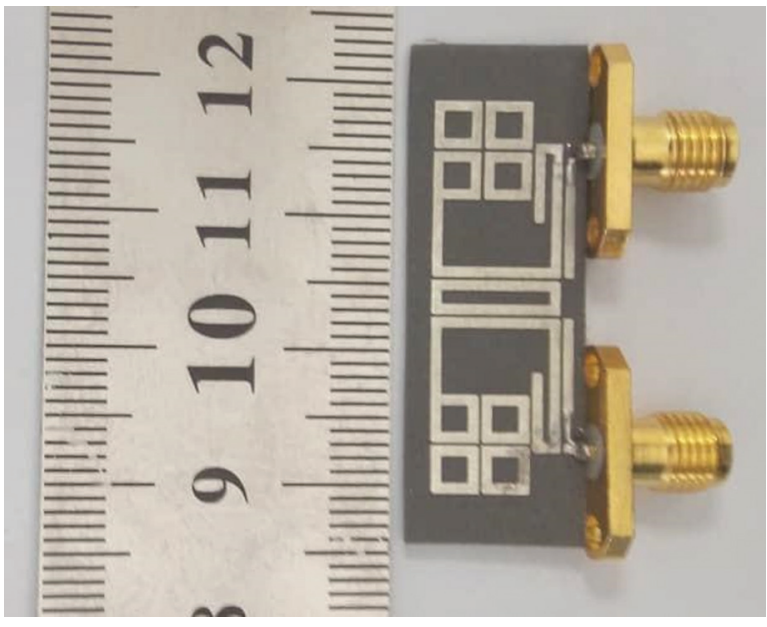

Fig. 8. Fabricated dual-band bandpass filter.
$3 \mathrm{GHz}$ is common between ultra-high-frequency and super high-frequency bands, it can be used in these applications. Fig. 7c shows the simulated wideband frequency response in the range of $0.1 \mathrm{GHz}-90 \mathrm{GHz}$. From Fig. 7c, it can be seen that the harmonic levels are $<-17 \mathrm{~dB}$ up to $85 \mathrm{GHz}$. Both return losses are better than $35 \mathrm{~dB}$, where the insertion losses are $0.3 \mathrm{~dB}$ and $1.3 \mathrm{~dB}$ at $3 \mathrm{GHz}$ and $6.3 \mathrm{GHz}$, respectively. The size of the proposed DB-BPF is $26.5 \mathrm{~mm} \times 12.5 \mathrm{~mm}$ $\left(0.35 \lambda_{\mathrm{g}} \times 0.16 \lambda_{\mathrm{g}}\right)$. Fig. 8 shows the fabricated DB-BPF.

According to the frequency response, it has several transmission zeros (TZs) which improved the frequency response. Due to the $\mathrm{TZ}$ of $-65 \mathrm{~dB}$ at $3.7 \mathrm{GHz}$, a good isolation between two passbands is obtained. Several TZs are created at $7.3,13,15.7,19$, and $23 \mathrm{GHz}$ with the attenuation levels of $-40,-40,-30,-38$, and $-58 \mathrm{~dB}$, respectively. Below the first channel, a TZ of $-82 \mathrm{~dB}$ is achieved at 1

TABLE I

Comparison with the Previous Works.

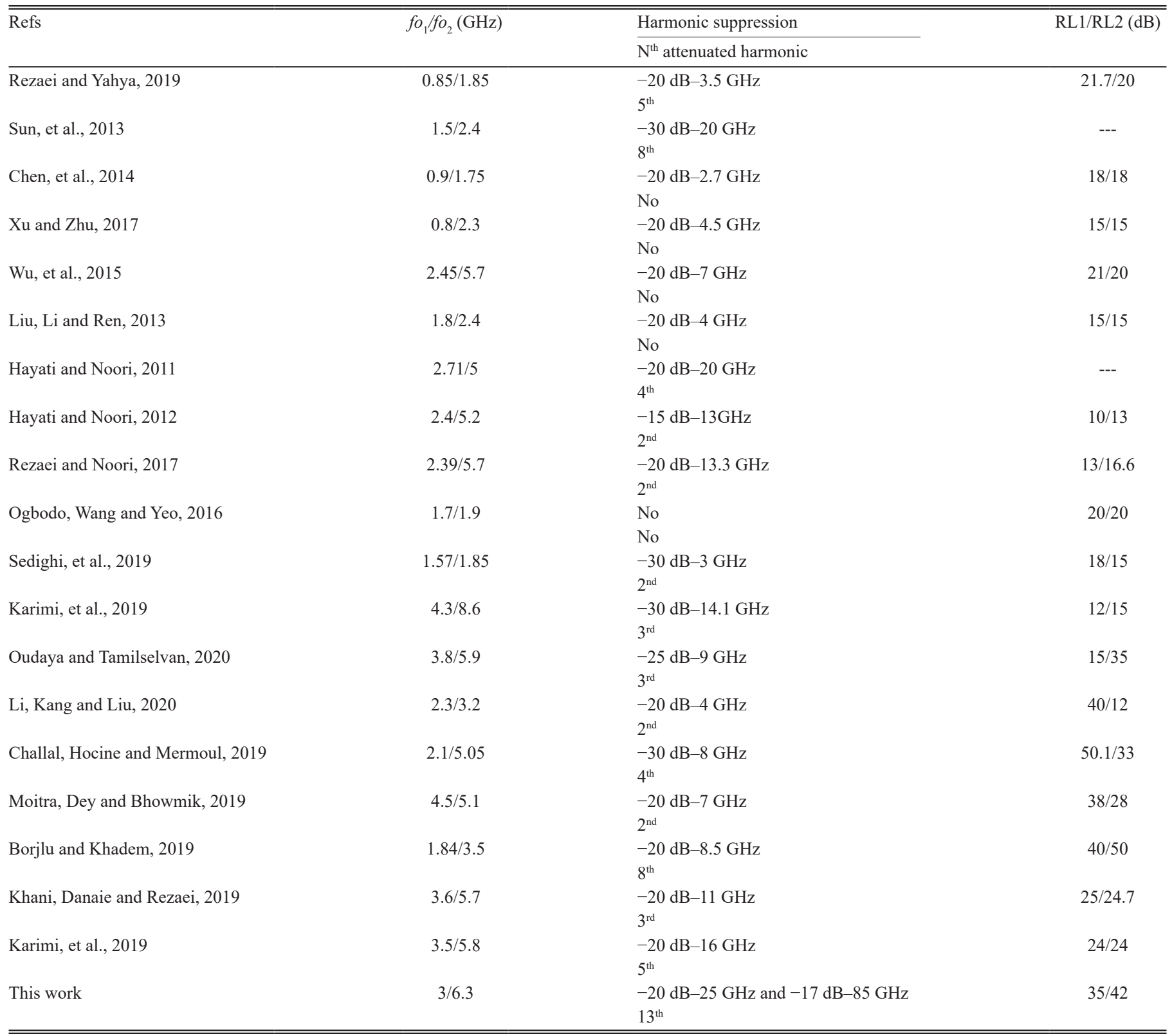


GHz. Meanwhile, we can see good selectivity at both channels. The comparison results with the previous DB-BPFs with various structures are shown in Table 1. The return losses at the first and second bands are presented by RL1 and RL2, respectively. According to the comparison table, the best harmonic attenuation and good return losses are obtained in this work.

\section{CONCLUSION}

We fabricated and measured a DB-BPF for ultra-wide stopband and multiservice applications. The proposed structure consists of closed loops and coupled lines. The designing method is based on analyzing the $L C$ approximated model of the proposed resonator, where a novel $L C$ model of coupled lines is presented. Moreover, a theoretical method is presented to attenuate the harmonics. The presented DBBPF has high selectivity and a wide stopband. The levels of harmonics are $<-17 \mathrm{~dB}$ up to $85 \mathrm{GHz}$. Moreover, it has other advantages in terms of high isolation between two channels, compact size and good return losses compared to the previous DB-BPFs.

\section{REFERENCES}

Badiaa, A.A., Otman, A., Azzeddin, N.R. and Ana Vazquez, A., 2020. Miniaturized multi-band stopband filter using circular split ring resonator and null gap separations between all parallel lines. Progress in Electromagnetics Research C, 106, pp.137-150.

Borjlu, S.R. and Khadem, M.S., 2019. Novel microstrip dual-band quad-section bandpass filter using radial stub for wireless communication. Journal of Electrical Engineering and Technology, 14, pp.1327-1333.

Challal, M., Hocine, K. and Mermoul, A., 2019. A novel design of compact dual-band bandpass filter for wireless communication systems. Wireless Personal Communications, 109, pp.1713-1726.

Chen, C.F., Chang, S.F., Tseng, B.H. and Weng, J.H., 2014. Compact dual-band stepped-impedance resonator filter with separate coupling paths. Electronics Letters, 50(21), pp.1551-1552.

Feng, W., Gao, X. and Che, W., 2015. Bandpass filters with improved selectivity based on dual-mode ring resonators. Progress in Electromagnetics Research Letters, 56, pp.1-7.

Hammed, R.T., 2015. Miniaturized dual-band bandpass filter using E-shape microstrip structure. AEU-International Journal of Electronics and Communications, 69, pp.1667-1672.

Hayati, M. and Noori, L., 2011. Compact dual band bandpass filter using open loop resonator loaded by in-line beeline for wideband applications. IEICE Electronics Express, 8(21), pp.1789-1794.

Hayati, M. and Noori, L., 2011. Compact dual-band band-pass filter with ultrawide stopband using open-loop resonator loaded by T-shape and open stubs. IEICE ELEX, 8(14), pp.1168-1173.

Hayati, M. and Noori, L., 2012. Compact dual-band band-pass filter using open loop resonator for multimode WLANs. Electronics Letters, 48(10), pp.573-574.

Hong, J.S. and Lancaster, M.J., 2001. Microwave Filter for RF/Microwave Application, John Wiley \& Sons, New York, USA.

Hsu, W., Lyu, P. and Chang, S., 2020. Design of a miniature dual-band band-pass filter with interlocked stepped-impedance resonators for $5 \mathrm{G}$ new radio access technology. International Journal of Microwave and Wireless Technologies, 12(8), pp.733-737.
Huang, D. and Huang, Z., 2014. Design of dual-band band-pass filter using dual-mode defected stub loaded resonator. Journal of Electrical and Computer Engineering, 2014, p.176560.

Karimi, G., Amirian, M., Lalbakhsh, A. and Ranjbar, M., 2019. A new microstrip coupling system for realization of a differential dual-band band-pass filter. $A E U$ International Journal of Electronics and Communications, 99, pp.186-192.

Karimi, G., Pourasad, Y., Lalbakhsh, A., Siahkamari, H. and Mohamadzade, B., 2019. Design of a compact ultra-narrow band dual band filter for WiMAX application. AEU-International Journal of Electronics and Communications, 110, p.152827.

Khani, S., Danaie, M. and Rezaei, P., 2019. Miniaturized microstrip dual-band bandpass filter with wide upper stop-band bandwidth. Analog Integrated Circuits and Signal Processing, 98, pp.367-376.

Li, D., Zhang, Y., Feng, X., Song, K. and Fan, Y., 2015. Dual-band band-pass filter with controllable center frequency and bandwidth using short stubloaded SIR and tri-section SIR. AEU-International Journal of Electronics and Communications, 69, pp.1004-1009.

Li, K., Kang, G. and Liu, H., 2020. High-selectivity adjustable dual-band band-pass filter using a quantic-mode resonator. Microsystem Technologies, 26, pp.913-916.

Liu, H.W., Li, S. and Ren, B.P., 2013. Microstrip dual-band band-pass filter with E-shaped multimode resonator. Electronics Letters, 49(14), pp.887-888.

Lu, H., Wu, W., Huang, J., Zhang, X. and Yuan, N., 2017. Compact dualmode microstrip bandpass filter based on greek-cross fractal resonator. Radioengineering, 26(1), pp.275-284.

Malherbe, J.A.G., 2016. An asymmetrical dual band band-pass filter. Microwave and Optical Technology Letters, 59(1), pp.163-168.

Moitra, S., Dey, R. and Bhowmik, P.S., 2019. Design and band coalition of dual band microstrip filter using DGS, coupled line structures and series inductive metallic vias. Analog Integrated Circuits and Signal Processing, 101, pp.77-88.

Ogbodo, E.A., Wang, Y. and Yeo, K.S.K., 2016. Microstrip dual-band bandpass filter using U-Shaped resonators. Progress in Electromagnetics Research Letters, 59, pp.1-6.

Oudaya, C.S. and Tamilselvan, S., 2020. A compact conductor-backed CPWbased dual band-pass filter for satellite S-band and C-band. Journal of Electrical Systems and Information Technology, 7(5), pp.2-11.

Pan, W.Q., Zhao, X.L., Zhang, Y. and Xu, J.X., 2015. High selectivity dual-band band-pass filter with tunable lower pass-band. International Journal of Antennas and Propagation, 2015, p.762504.

Rezaei, A. and Noori, L., 2017. Design of microstrip wide stopband quad-band bandpass filters for multi-service communication systems. AEU-International Journal of Electronics and Communications, 81, pp.1388-1393.

Rezaei, A. and Noori, L., 2017. Tunable microstrip dual-band band-pass filter for WLAN applications. Turkish Journal of Electrical Engineering and Computer Sciences, 25, pp.1388-1393.

Rezaei, A. and Yahya, S.I., 2019. High-performance ultra-compact dual-band band-pass filter for global system for mobile communication-850/global system for mobile communication-1900 applications. ARO-The Scientific Journal of Koya University, 7(2), pp.34-37.

Rezaei, A., Yahya, S.I. and Jamaluddin, M.H., 2020. A novel microstrip diplexer with compact size and high isolation for GSM applications. AEU-International Journal of Electronics and Communications, 114, p.153018.

Rezaei, A., Yahya, S.I., Noori, L. and Jamaluddin, M.H., 2019. Design and fabrication of a novel compact low-loss microstrip diplexer for WCDMA and WiMAX applications. Journal of Microwaves, Optoelectronics and Electromagnetic Applications, 18(4), pp.482-491.

Rezaei, A., Yahya, S.I., Nouri, L. and Jamaluddin, M.H., 2020. Design of a low-loss microstrip diplexer with a compact size based on coupled meandrous 
open-loop resonators. Analog Integrated Circuits and Signal Processing, 102, pp.579-584.

Salleh, M.K.M., Prigent, G., Pigaglio, O. and Crampagne, R., 2008. Quarterwavelength side-coupled ring resonator for band-pass filters. IEEE Transactions on Microwave Theory and Techniques, 56(1), pp.156-162.

Sedighi, M.S., Dousti, M., Dolatshahi, M. and Ghalamkari, B., 2019. Tunable dual-band bandpass filter for multi-standard applications. AEU-International Journal of Electronics and Communications, 111, p.152885.

Sun, S.J., Su, T., Deng, K., Wu, B. and Liang, C.H., 2013. Compact microstrip dual-band band-pass filter using a novel stub-loaded quad-mode resonator. IEEE Microwave and Wireless Components Letters, 23(9), pp.465-467.

Wu, G.C., Wang, G., Liang, J.G., Gao, X.J. and Zhu, L., 2015. Miniaturized microstrip dual-band band-pass filter using novel symmetric double-spiral resonators for WLAN application. Electronics Letters, 51(15), pp.1177-1178.

Wu, H.W., Su, Y.K., Weng, M.H. and Yang, R.Y., 2008. Design of dual-band band-pass filter using diverse quarter wavelength resonator for GPS/WLAN Applications. Microwave and Optical Technology Letters, 50(10), pp.2694-2696.

Xu, J. and Zhu, Y., 2017. Compact semi-lumped dual-and tri-wideband band-pass filters. IET Microwaves Antennas and Propagation, 11(1), pp.53-58.
Yahya, S.I., Rezaei, A. and Nouri, L., 2020a. Design and performance of microstrip diplexers: A review. ARO-The Scientific Journal of Koya University, 8(1), pp.38-49.

Yahya, S.I., Rezaei, A. and Nouri, L., 2020b. Design and fabrication of a high-performance microstrip multiplexer using computational intelligence for multi-band RF wireless communications systems. AEU-International Journal of Electronics and Communications, 120, p.153190.

Yahya, S.I., Rezaei, A. and Nouri, L., 2020c. A novel miniaturized microstrip lowpass-band-pass diplexer using patch and interdigital cells for wireless networks. AEU-International Journal of Electronics and Communications, 126(153404), pp.1-7.

Yang, R.Y., Hon, K., Hung, C.Y. and Ye, C.S., 2010. Design of dual-band bandpass filters using a dual feeding structure and embedded uniform impedance resonators. Progress in Electromagnetics Research, 105, pp.93-102.

Zhu, H. and Abbosh, A.M., 2016. Single-and dual-band band-pass filters using coupled stepped-impedance resonators with embedded coupled-lines. IEEE Microwave and Wireless Components Letters, 26(9), pp.675-677.

Zong, B.F., Wang, G.M., Zeng, H.Y. and Wang, Y.W., 2012. Compact and high performance dual-band band-pass filter using resonator-embedded scheme for WLANs. Radioengineering, 21(4), pp.1050-1053 